\title{
Lens-specific expression of recombinant ricin induces developmental defects in the eyes of transgenic mice
}

\author{
Carlisle P. Landel, Jun Zhao, Dean Bok, ${ }^{1}$ and Glen A. Evans ${ }^{2}$ \\ Molecular Genetics Laboratory; The Salk Institute for Biological Studies, La Jolla, California 92037 USA; ${ }^{1}$ Jules Stein Eye \\ Institute, University of California at Los Angeles, Los Angeles, California 90024 USA
}

\begin{abstract}
An expression system for cell lineage ablation in transgenic mice was constructed in which a modified form of the A subunit of ricin, a toxic lectin produced by the castor bean Ricinus communis, can be expressed under the direction of tissue-specific regulatory signals. A chimeric gene was formed by fusing the promoter and 5'flanking sequences of the lens-specific mouse $\alpha A$-crystallin gene with a modified ricin A CDNA, and this construction was integrated into the germ line of transgenic mice. These animals develop profound microphthalmia with severe developmental defects of the eye, relating primarily to the disorganization and death of cells forming the lens. In addition, this defect is associated with several abnormalities, including eye size, folding of the retina, and ectopic lens material in other regions of the eye. The phenotype of this engineered developmental mutation suggests that the normal development of $\alpha \mathrm{A}$-crystallin-producing lens fiber cells is essential for the proper growth, organization, and orientation of optic structures.
\end{abstract}

[Key Words: Transgenic mice; ricin; crystallin; eye development]

Received May 12, 1988; revised version accepted July 8, 1988.

The molecular processes regulating cellular determination during organogenesis are a poorly understood aspect of mammalian developmental biology. In many complex organ systems, including the immune and nervous systems, a large number of phenotypically distinct cell types are derived from a relatively small number of progenitor cells through successive waves of cell proliferation and differentiation. The interactions of cells of different lineages, in concert with environmental or genetically programmed cues, may guide the development of complex morphologic patterns and the initiation and maintenance of organogenesis. These developmental patterns may be studied readily in some organisms where powerful tools are available. However, in vertebrates, particularly mammals, only certain cell lineages permit experimental access for the study of cellular determination and morphogenesis.

A number of techniques have been developed for examining cell lineage relationships in vertebrates. Cellmarking techniques, utilizing injected intracellular tracers (Jacobsen 1981; Kimmel and Warga 1986), genetic markers (Herrup 1986), or injections of retroviruses expressing histological markers (Price et al. 1987; Turner and Cepko 1987), permit the progeny from one or more marked progenitors to be identified and lineage relationships determined. Cell ablation techniques, using classical surgical approaches (Roux 1888), laser microbeams (Sulston and White 1980), drugs (Burns et al. 1983), or genetic mutations (Horvitz et al. 1983), allow

${ }^{2}$ Corresponding author. the elimination of progenitor cells and the resulting cellular progeny. Recently, the exquisite specificity of developmentally controlled gene expression has been exploited for genetic cell ablation in transgenic mice. With this approach, the expression of a toxic compound or metabolite can be induced at a specific time during cellular differentiation, resulting in cellular 'suicide.' Palmiter et al. (1987), expressed the toxic A subunit of diphtheria toxin under the direction of the regulatory sequences of the mouse pancreatic elastase gene, leading to ablation of the neonatal pancreas. Breitman et al. (1987) induced microphthalmia in transgenic mice by expressing diphtheria toxin from the $\gamma 2$-crystallin promoter in mouse lens. However, the $\gamma$-crystallins are expressed only in lens fiber cells and not in the epithelial cells that serve as their progenitors. Also, $\gamma$-crystallins are expressed maximally during early lens development and only at minimal levels thereafter. Thus, ablation directed by a $\gamma$-crystallin promoter might be expected to induce only minimal perturbations of eye development.

In this study, we developed a cell lineage ablation system where the toxic A subunit of the lectin ricin can be expressed from cell-specific regulatory sequences. Ricin is produced by the castor bean Ricinus communis as a nontoxic precursor preproricin that is processed and activated by protease activity (Olsnes and Phil 1982). Mature ricin consists of a 262-amino-acid B polypeptide, containing galactose-specific cell-surface binding and membrane translocation activity, disulfide cross-linked to a 267 -amino-acid toxic A subunit, which is a $\mathrm{N}$-glycosidase specific for $\mathrm{A}_{4324}$ of ribosomal 28S RNA (Endo and 
Tsurugi 1987). Internalization of the A subunit, mediated by cell-surface binding of the B subunit, catalytically destroys ribosome function and blocks protein synthesis. In the absence of the binding and internalization functions inherent in the B subunit, the ricin A subunit remains in the extracellular space where its toxicity is extremely low (Pastan et al. 1986). This property is important for the precision of genetic ablation in transgenic mice, because the release of endogenously synthesized A chain during genetically programmed cell death will not kill surrounding cells.

Vertebrate lens provides an ideal system for the development of toxigenic gene systems because the expression of toxic genes in developing lens fiber cells results in readily apparent structural abnormalities that are nonlethal and associated with little systemic pathology (Breitman et al. 1987). Mammalian lens cells express three families of crystallin genes, $\alpha, \beta$, and $\gamma$, which are coordinately regulated and expressed in a precisely regulated temporal pattern (Van Leen et al. 1987a). The murine $\alpha$-crystallin family consists of two genes, $\alpha \mathrm{A}$ and $\alpha \mathrm{B}$. The $\alpha \mathrm{A}$-crystallin gene produces two polypeptides by alternate mRNA splicing, $\alpha \mathrm{A} 2$ and $\alpha \mathrm{A}^{\text {ins }}$, first detected at day 10 of embryogenesis (King and Piatigorski 1983), whereas $\beta$ - and $\gamma$-crystallins appear later at days 15-17 (Bloemendal 1982). In the mouse, $\alpha$-crystallins are first detectable at day $11, \gamma$-crystallins between day 11.5 and 12 , and $\beta$-crystallins at day 12.5 of embryogenesis (Zwaan and Silver 1983). The promoter and 5'flanking sequences of the $\alpha \mathrm{A}$-crystallin gene appear to include the majority of the significant regulatory signals for cell-specific and developmentally regulated gene expression (Chepelinsky et al. 1987) and function appropriately in transgenic mice (Overbeek et al. 1985; Mahon et al. 1987).

We induced the expression of a modified but highly toxic ricin A subunit from the promoter and $5^{\prime}$-flanking region of the $\alpha \mathrm{A}$-crystallin gene in transgenic mice. Eye development in the presence of this gene results in a dis- tinct, homogeneous microphthalmic phenotype characterized by highly disorganized lens fiber cells and failure of the lens to develop normally. In addition, this phenotype is associated with several surprising abnormalities, including distinctive developmental abnormalities of the neurosensory retina and the appearance of ectopic lens fiber cells in the eye. These abnormalities define important developmental interactions between structures arising separately from mesoderm, surface ectoderm, and neuroectoderm in the vertebrate visual system.

\section{Results \\ Construction of a ricin A toxigene}

To produce a chimeric gene that would express only the A chain of ricin and generate a gene product toxic only to the cells in which it was expressed, a restriction fragment containing the region encoding the entire A subunit was removed from the preproricin cDNA construct pUC8RA (Lamb et al. 1985) and fused to the promoter and $5^{\prime}$-flanking region of the mouse $\alpha \mathrm{A}$-crystallin gene (Chepelinsky et al. 1987). A synthetic oligonucleotide containing the sequence AATAAA was inserted downstream to supply a polyadenylation signal (Fig. 1). The toxin produced by this construction is thus a modified form of ricin A containing 24 additional amino acids at the amino terminus, which do not act as a true signal peptide. Eighteen additional amino acids are present at the carboxyl terminus, consisting of the 12 amino acids of the connecting peptide and 6 amino acids from the amino terminus of the $B$ chain. The resulting modified ricin A chain demonstrates a high degree of toxicity when expressed in yeast, mammalian cell-free extracts and after transfection into eukaryotic cell lines in culture. However, these experiments suggest that this gene product may be somewhat attenuated in toxic activity when compared to the natural ricin A chain (data not shown).

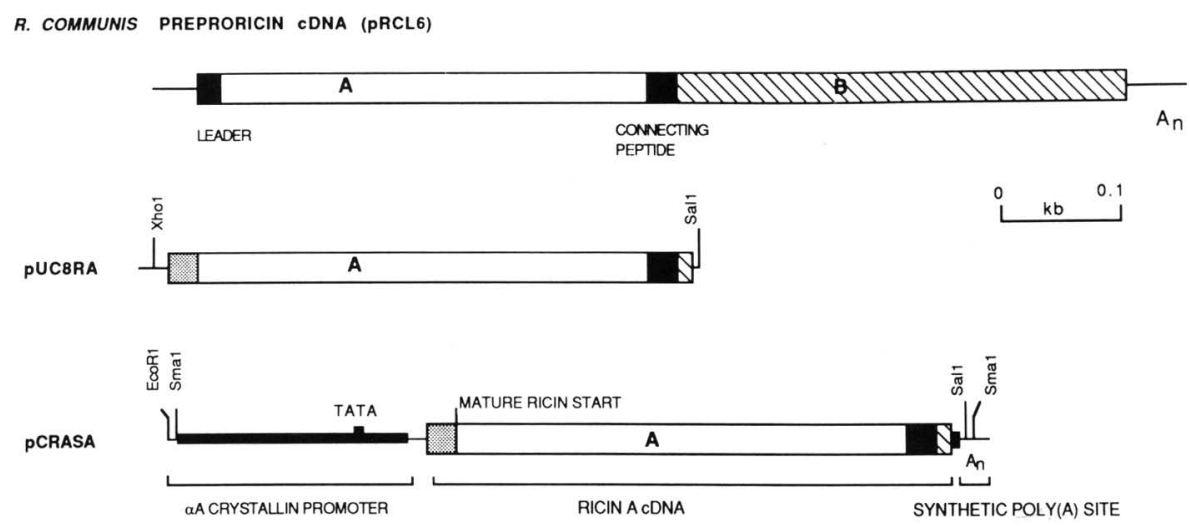

Figure 1. Construction of a gene for the intracellular expression of the A subunit of ricin in transgenic mice. The mature ricin molecule is synthesized as a precursor, preproricin, containing A and B subunits linked by a connecting peptide. An XhoI-SaII restriction fragment containing the majority of the A subunit sequence was excised and linked to a BglII-BamHI fragment containing the mouse $\alpha \mathrm{A}$-crystallin promoter using the polylinker of plasmid pUC19. A poly(A) addition signal was added at the $3^{\prime}$ end by inserting a synthetic oligonucleotide containing the sequence AATAAA, as well as a SmaI restriction site. Restriction fragments used as hybridization probes are underlined. 

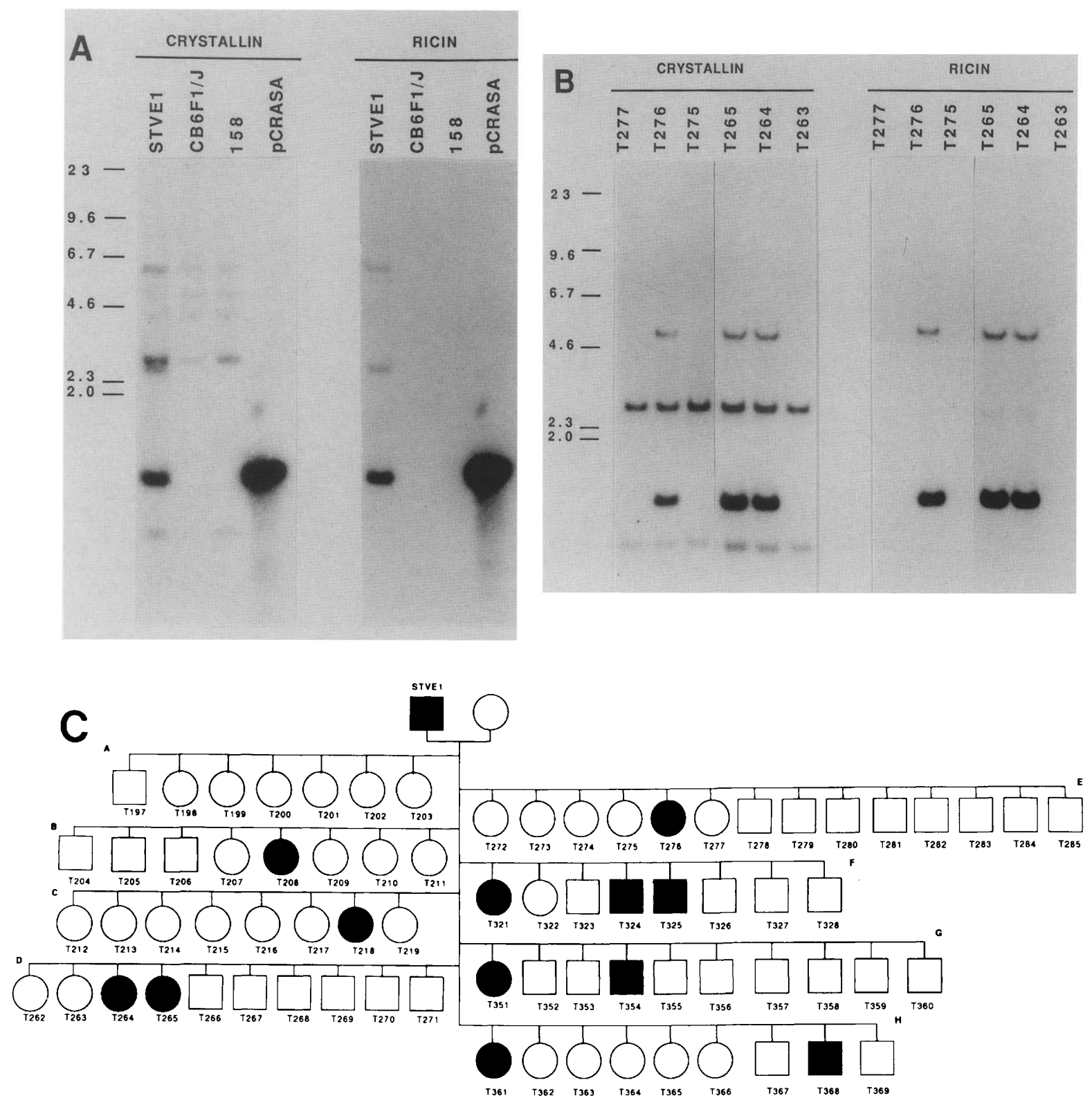

Figure 2. Transgenic mice carrying the $\alpha$ A-crystallin/ricin A fusion gene isolated from plasmid pCRASA. DNA isolated from tails was digested with $E c o R I$, which does not cut within the transgene sequence, and hybridized to an $\alpha \mathrm{A}$-crystallin probe or a ricin A probe. (A) Southern blot of tail DNA from a founder transgenic mouse (STVE1), normal control mouse (CB6F1/J), nontransgenic littermate (158), and plasmid DNA containing the fusion gene (pCRASA). (B) Southern blot of tail DNA from Fl progeny transgenic mice (T276, T265, and T264) and nontransgenic littermates \{T277, T275, and T263). Size markers are HindIII restriction fragments of bacteriophage $\lambda .|C|$ Pedigree showing the inheritance of the $\alpha$ A-crystallin/ricin sequences in the first eight litters of male founder STVE1. All mice that inherited the ricin transgene (black symbols) also demonstrated the microphthalmic phenotype.

\section{Expression of the ricin toxigene in transgenic mice}

To induce developmentally controlled death of the $\alpha \mathrm{A}$ crystallin-producing lens fiber cells, the fusion gene was excised from the plasmid with SmaI, separated from the vector sequences by gel electrophoresis, and microinjected into the male pronucleus of fertilized mouse eggs. Isolated mouse embryos were injected at the one-cell stage and implanted into pseudopregnant foster recipients, resulting in 85 live births. Southern blot analysis of tail DNA prepared from 3-week-old weanlings was carried out to detect transgenic animals in which the hybrid gene, including both crystallin and ricin sequences, was integrated into the genome (Fig. 2A,B). Three weanlings had transgenic sequences integrated into the genome, as evidenced by analysis of tail DNA. A comparison of the size of the intact fusion gene with the fragment isolated from plasmid DNA (Fig. 2A) demonstrated that the transgene sequences were not rearranged in the genome. The success rate of producing transgenic animals with this construction was $3.5 \%$, as 
opposed to a consistent $25-30 \%$ with other intact genes and fusion genes (S. Chen, C.P. Landel, J. Zhao, and G.A. Evans, unpubl.). This significantly lower rate of transgenic animal production may be the result of transient expression of the ricin fusion gene immediately after injection into embryos, resulting in fewer total viable embryos. However, resulting litter sizes in these transgenic experiments were normal, and the culture of isolated embryos to the two-cell stage after injection with the $\alpha \mathrm{A}$-crystallin/ricin A fusion gene did not reveal inordinately high levels of embryo death. This finding is not surprising, as protein synthesis is not required for the initiation of the first cell cleavage during mammalian embryogenesis, and it seems likely that the low-level expression of the toxic gene product at some point in early embryogenesis, perhaps after implantation, results in the decreased frequency of resulting transgenic animals.

All of the mice with the $\alpha \mathrm{A}$-crystallin/ricin A transgene integrated in the genome demonstrated a marked developmental abnormality that was observed easily at 2-3 weeks of age and, indeed, before DNA analysis could be carried out. These animals suffered marked microphthalmia, and the very small eyes did not protrude from the eye cavity. Visual examination at 2 weeks revealed that the eyes were much smaller than normal, and this abnormality became more profound with age. At 25 days after birth, the eyes were so small as to remain closed most of the time (Fig. 3).

Only one founder mouse was fertile and was used to establish a strain of transgenic mice by backcrossing with CB6F2/J mice (Fig. 2C). This individual demonstrated a microopthalmic phenotype and carried 5-10 copies of the integrated $\alpha \mathrm{A}$-crystallin/ricin A transgene (Fig. 2B). Litter sizes were normal, and reproductive capacity was otherwise identical with that seen for other transgenic strains and normal mouse strains. As expected, among litters from a transgenic founder mated with a nontransgenic host, F1 transgenic animals could be identified by very early, subtle abnormalities of the face in the area of the eyes. This area, which is normally slightly convex due to the underlying eyeball, was flat in transgenic animals. Within a few days after eye opening, unequivocal abnormalities were easily visible in the eyes of these animals and were confirmed several weeks later by analysis of DNA isolated from tails (Fig. 2B). By 3 weeks after birth, transgenic animals were easily distinguished from normal littermates by the appearance of the flat, nearly closed eyes. Sixteen percent of the offspring from this founder male were transgenic, suggesting that this animal may be mosaic in the germ line. This is consistent with the observation that the F2 generation derived from this founder (not shown) transmitted the defect with Mendelian inheritance. In all cases, perfect correlation was observed between the observable phenotypic abnormalities in eye structure and the presence of the transgene (Fig. 2C). We have not observed any animals containing this transgene that do not demonstrate an identical morphology and behavior; and with the exception of a single mosaic founder animal, the defect is seen symmetrically in both eyes.

\section{Toxin-induced alterations in lens structure}

Eyes from founder animals, Fl progeny, and control nontransgenic littermates were removed for histological analysis at 3 or 6 weeks of age, fixed using techniques that preserve lens structure, embedded in plastic, and sectioned. Normal eyes (Fig. 4A) have a large lens with a poorly fixed center and a uniformly stained periphery. The retina is a thin stratified layer of cells at the posterior surface, the iris is well defined, and much of the inner volume of the eye is occupied by the lens. In contrast, the eyes from $\alpha \mathrm{A}$-crystallin/ricin A transgenic /Fig. 4B-D) are one-third to one-fourth normal size, and their lens are vacuolated and disorganized in structure. The vitreous chambers are decreased in size and nearly filled by the neural retina. Lens epithelial cells, the lens bow, and successive layers of flattened fibrous secondary lens cells are apparent in the normal lens at 3 weeks of age (Fig. 5A), and fiber cell nuclei are primarily concentrated at the peripheral lens bow. The abnormal lens of the transgenic mice contained one or more fluid-filled cavities (Figs. 4C and 7A). The lens epithelium, usually limited to the anterior portion of the lens, appeared normal. The lens fiber cells in these transgenic mice were much more disorganized than those seen in normal eyes and failed to flatten and form thin fibrous layers (Fig. 5B). The cells appeared spheroid or elongated and less intensely stained, perhaps indicating various crystallin densities. Cell nuclei were no longer neatly organized into a bow-like array characteristic of the normal lens.

\section{Lens-specific ricin expression induces abnormalities of the retina}

Lens-specific abnormalities could, for the most part, be attributed to ablation of early $\alpha \mathrm{A}$-crystallin producing cells and were consistently found in all transgenic mice examined. However, in addition to the expected lens abnormalities, a defect in development of the neurosensory retina was observed as a consistent feature of this transgenic phenotype. The normal retina is a highly organized, layered structure that lines the posterior inner surface of the eye (Fig. 6A). The transgenic animals carrying $\alpha \mathrm{A}$-crystallin/ricin $\mathrm{A}$ gene were found to have the

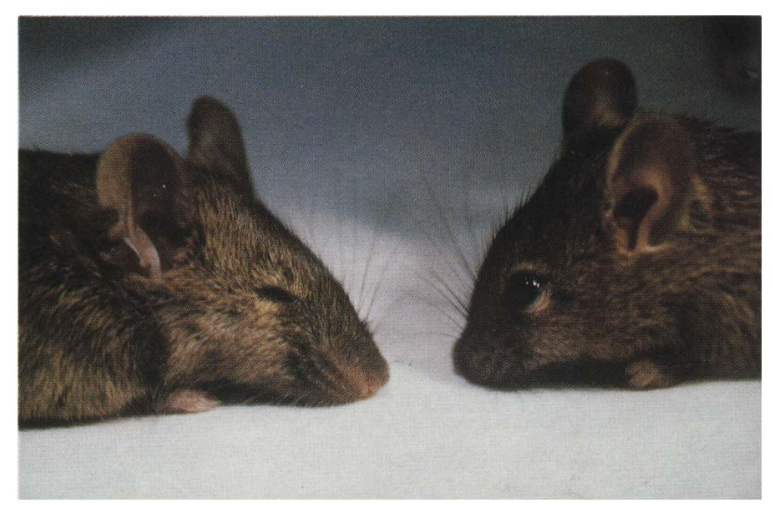

Figure 3. Transgenic mouse displaying ocular dysmorphology at 6 weeks of age (left) compared to a control littermate (right). 

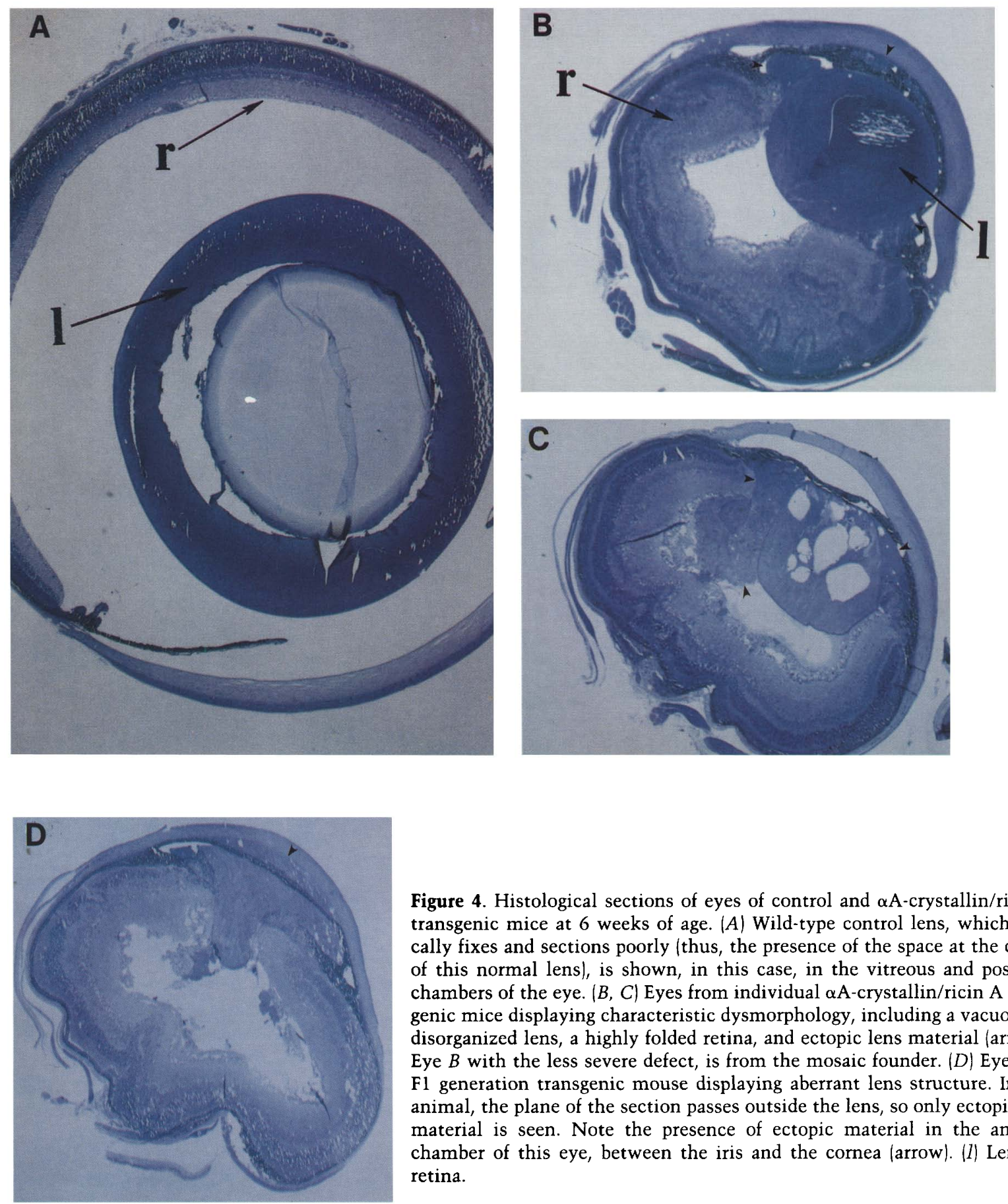

Figure 4. Histological sections of eyes of control and $\alpha$ A-crystallin/ricin A transgenic mice at 6 weeks of age. (A) Wild-type control lens, which typically fixes and sections poorly (thus, the presence of the space at the center of this normal lens), is shown, in this case, in the vitreous and posterior chambers of the eye. $(B, C)$ Eyes from individual $\alpha \mathrm{A}$-crystallin/ricin A transgenic mice displaying characteristic dysmorphology, including a vacuolated, disorganized lens, a highly folded retina, and ectopic lens material (arrows). Eye $B$ with the less severe defect, is from the mosaic founder. $(D)$ Eye from F1 generation transgenic mouse displaying aberrant lens structure. In this animal, the plane of the section passes outside the lens, so only ectopic lens material is seen. Note the presence of ectopic material in the anterior chamber of this eye, between the iris and the cornea (arrow). (1) Lens; $(r \mid$ retina.

posterior chamber filled almost entirely with folded neural retinal layers. The normal inner and outer nuclear layers, photoreceptor inner and outer segments, and ganglion cell layers appeared to be present but were folded in a disorganized pattern (Figs. 4B-D, and 6B). In addition, the optic nerve, which contains all of the retinal ganglion cell axons, was significantly reduced in size (not shown). It is likely that this retinal abnormality is associated with severely defective vision and is presumably the result of neural retinal growth in the face of severely limited space for expansion during embryo- genesis. This retinal malformation suggests that development of structures arising separately from surface ectoderm and neuroectoderm are codependent for accurate morphogenesis.

Ricin expression is associated with the appearance of ectopic crystallin lens

A second abnormality found in eyes of all transgenic animals examined was the appearance of ectopic lens material present outside of the normal lens capsule (Fig. 7). 
This material was observed in the angle between iris and ciliary body (posterior chamber), between the anterior iris and cornea (anterior chamber), and in the vitreous chamber extending to the neural retina. This material was seen as collections of cells with a staining pattern and morphology similar to that of the abnormal fiber cells seen inside the lens. These ectopic lentoid cells also demonstrated a staining pattern less uniform than normal lens and had a structural disorganization similar to that found in the lens fiber cells in the transgenic lens. Clustered groups of these ectopic cells were bounded by a basement membrane. As with the lens cells, these ectopic cells had a disorganized appearance and failed to flatten to form the fibrous lentoid layers characteristic of normal lens. To our knowledge, this type of abnormality has not been observed with other genetic or induced aberrations of the mammalian eye or lens.

Additional analysis of crystallin/ricin transgenic mice, including pathological analysis of other tissues such as brain, kidney, liver, intestine, thymus, spleen, muscle, and heart, did not reveal any additional pathological abnormalities. Aside from the possibility of subtle neurological abnormalities, the phenotype of these transgenic mice is restricted to malformations in the eye, neurosensory retina, and optic nerve. Thus, the crystallin/ricin transgene induces a dominantly inherited phenotype with complete penetrance and includes (1) severe microphthalmia, (2) malformation of lens with central lens vacuolization, (3) disorganized lens fiber cells, (4) retinal folding, and (5) ectopic lentoid material.

\section{Discussion \\ Toxigenic ablation disrupts normal morphogenesis}

The eye is a precise optical system that requires carefully coordinated morphogenesis of a number of distinct structures for proper function. The vertebrate lens begins to develop when a small population of epithelial cells forms the lens placode under the inductive influence of the underlying optic vesicle. The lens placode then invaginates to form the lens vesicle, a hollow sphere of epithelial cells surrounded by a basement membrane. Under the inductive influence of the retina, cells of the posterior hemisphere elongate into primary lens fibers, fill the lumen of the vesicle, and eventually contact the epithelium forming the embryonic lens nucleus. Further lens growth is accomplished through the differentiation of epithelial cells along the anterior-posterior equatorial plane into secondary lens fibers that elongate to surround the nucleus. The lens continues to grow throughout life by the accumulation of additional fiber cells at the periphery, whereas the fiber cells at the center of the lens become metabolically quiescent. The mature lens is thus a spheroid with primary lens fibers surrounded by layers of columnar secondary lens fibers, bounded anteriorly by a hemispherical monolayer of epithelial cells, and encapsulated by a basement membrane.
In mammals, lens fiber cells synthesize $\alpha-, \beta-$, and $\gamma$ crystallins through gene expression that is regulated temporally and spatially in lens development (Bloemendal 1982). The $\alpha$ A-crystallins are the earliest detectable crystallins in murine lens development, initially detectable by immunofluorescence in cells at the posterior invaginating lens placode at day 10-11 of development (Zwaan and Silver 1983). When the primary lens fibers elongate to fill the lumen of the lens vesicle at day 13-14 of embryogenesis, $\alpha \mathrm{A}$-crystallin expression can be detected in all the cells of the developing lens, including the anterior, nondividing epithelial cells (Zwaan 1975). In the rat, Northern blot analysis confirms that $\alpha \mathrm{A}$-crystallin first appears at the time of primary fiber differentiation (Van Leen et al. 1987a) and in situ hybridization demonstrates that $\alpha \mathrm{A}$-crystallin is found in lens fiber cells and in the anterior epithelial cells (Van Leen et al. 1987b).

Studies of chick lens embryogenesis after extirpation of the developing lens have suggested that normal development of the living lens is critical for expansion and growth of the eye (Columbre and Columbre 1964). Transgenic mice expressing ricin under the control of crystallin regulatory sequences would be expected to suffer significant fiber cell death during lens embryogenesis. It is therefore not unexpected that genetically engineered death of portions of the developing lens leads to inhibition of eye growth. The characteristic and dramatically abnormal retinal morphology may be the result of expansion of the neurosensory retina in the absence of normal growth of the encapsulating eye wall, resulting in folding and compression of retinal structures within an abnormally small space.

Previous studies have suggested that $\alpha \mathrm{A}$-crystallins are expressed in both lens fiber and epithelial cells based on immunohistochemistry using $\alpha$-specific antisera [Zwaan 1975; Zwaan and Silver 1983)] Expression of chloramphenicol acetyltransferase (CAT) from an $\alpha \mathrm{A}$ crystallin promoter in transgenic mice demonstrated small amounts of CAT activity in microdissected lens epithelia (Overbeek et al. 1985). However, the lens of crystallin/ricin transgenic mice have a normal-appearing epithelial cell layer surrounding highly disorganized fiber cells. If ricin expression was induced in epithelial cells under the direction of the $\alpha \mathrm{A}$-crystallin promoter, one might expect an absent or severely disorganized eplithelial cell layer. One possibility is that lens epithelial cells may be totally or partially resistant to the toxic action of ricin. However, a more likely explanation, consistent with the results reported here, is that $\alpha \mathrm{A}$-crystallin is produced primarily in differentiating lens fiber cells and not expressed in most epithelial cells at the same developmental stage. The actual distribution of $\alpha \mathrm{A}$-crystallin among epithelial and fiber cells in developing mouse lens will require further investigation.

The cavities found in the center of the transgenic lens are likely the result of the failure of the majority of the primary fiber cells to fill the lumen of the lens vesicle. These cells begin to differentiate, undergo a small amount of growth while filling with some crystallin, 
and then die, leaving the population of small cells seen at the posterior of the lens. This death of fiber cells near the posterior epithelium, combined with the continued growth of the anterior epithelial layer, would cause the highly abnormal appearance of the lens bow. The lens dysmorphology seen in these transgenic mice could also be generated through an alternate mechanism, where the cavities in the lens arise as the result of the removal of cells killed by ricin. Expression of ricin in the earliest differentiating lens cells would be expected to cause cell death primarily in the oldest or most nuclear cells, resulting in the formation of fluid-filled vesicles at the lens core. The death of additional $\alpha \mathrm{A}$-crystallin-producing cells later in differentiation would result in severely disrupted lens architecture, perhaps because of recruitment of peripheral cells to replace the dying nuclear cells. However, the absence of visible macrophages in the transgenic lens suggests that the mechanism of dysmorphology is unlikely to require significant macrophage activity. These mechanisms could be distinguished by the examination of transgenic lens morphology during embryonic development. Alternatively, the surviving cells seen in the transgenic lens could represent cells that have failed to be killed by toxin expression (Breitman et al. 1987), and the disorganized morphology could result from proliferation of a small number of cells that have escaped toxin action.

\section{Ectopic lens formation may indicate in vivo transdifferentiation}

The ectopic lentoid material found consistently in all transgenic animals examined is a finding unique to those transgenic mice expressing the $\alpha$ A-crystallin/ricin A gene. This material may represent migration of differentiating epithelial or fiber cells to a new, extra-lentoid position as a result of induced degeneration of the differentiating lens epithelium. The examination of serial sections from several transgenic eyes revealed no evidence of a breakdown of the lens capsule, and histological evidence for a direct cellular extension between the lens and ectopic material could not be established. Thus, it is unlikely that migration of lens fiber or epithelial cells can explain this unexpected anatomical finding. However, the eye is one of the few tissues where evidence has accumulated for cellular transdifferentiation (an overt change in the differentiation phenotype) in amphibians and in avian and mammalian tissue culture systems (Okada 1980, 1983). In amphibians, retinal pigmented epithelial cells can transdifferentiate to lens fiber cells in culture and result in complete lens regeneration from the dorsal iris after extirpation or destruction of the normal lens. Avian neural retinal cells in culture can be induced to transdifferentiate to either crystallinproducing lens fiber cells or neurons under the influence of exogenous factors or altered culture conditions (Yasuda et al.1983; Reh et al.1987). Though further work is necessary to clarify the origin of the ectopic lentoid material found in these transgenic mice, the histological pattern of ectopic lens associated with epithelial or pigmented retinal cells would be consistent with the hypothesis that these cells result from the conversion of ciliary body, iris, or retinal cells to crystallin-producing lens fiber cells. These cells presumably accumulate some crystallin before toxin production results in cell death, leading to a collection of cells with a morphology very similar to that of the fiber cells within the transgenic lens. Additional studies combining lineage analysis by cell marking with programmed cell ablation should clarify the origin of the ectopic lens cells in these transgenic mice.

\section{Toxic ablation allows precise programmed perturbations in developing cell lineages}

Breitman et al. (1987) produced transgenic mice expressing a diphtheria toxin A chain from the $\gamma 2$-crystallin regulatory sequences and produced mice with a superficially similar, but much less severe, microphthalmia. However, the phenotype of these animals is distinct and different from that displayed by the $\alpha \mathrm{A}$ crystallin/ricin A transgenic mice described here. $\gamma 2$ Crystallin/diphtheria toxin transgenic mice demonstrate marked variations in the degree of microphthalmia, including asymmetry of the defect in a single individual. In the presence of this gene product, the degree of lens disorganization varies from strain to strain, between individuals of a single strain, and between the eyes of single individuals. The microphthalmia observed with $\gamma 2$-crystallin/diphtheria toxin transgenic mice is less severe than in $\alpha \mathrm{A}$-crystallin/ricin transgenic mice. Furthermore, the neurosensory retina in the $\gamma 2$-crystallin/diphtheria toxin mice has a normal morphology, and ectopic expression of lens crystallins in fiber-like cells outside of the lens capsule has not been reported in these animals. These differences in phenotypes are likely due to the specific differences in the spatial and temporal pattern $\alpha$-and $\gamma$-crystallin expression and the differentially programmed cell ablation by toxigenic expression. These studies suggest that quite precise genetic perturbations affecting cell lineage can be directed to the same cell types within a single developing organ system and result in distinct phenotypes and structural abnormalities.

Many naturally occurring mutations in mice affect normal development of the lens. Aphakia, (ak/ak) is an autosomal recessive mutation characterized by small, pupilless eyes that fail to bulge from the orbital cavity (Varnum and Stevens 1968). This mutation causes the failure of proper differentiation of the lens placode and results in abnormal lens growth and differentiation, reducing the size of the eye. This defect likely involves disruption of mitosis in dividing lens epithelial cells (Zwaan and Kirkland 1975) that subsequently fail to express crystallins (Zwaan 1975). In this mutation, like the $\alpha$ A-crystallin/ricin transgenic mice described here, folding of the neural retina that partially or completely fills the vitreous chamber is apparent. This observation, 

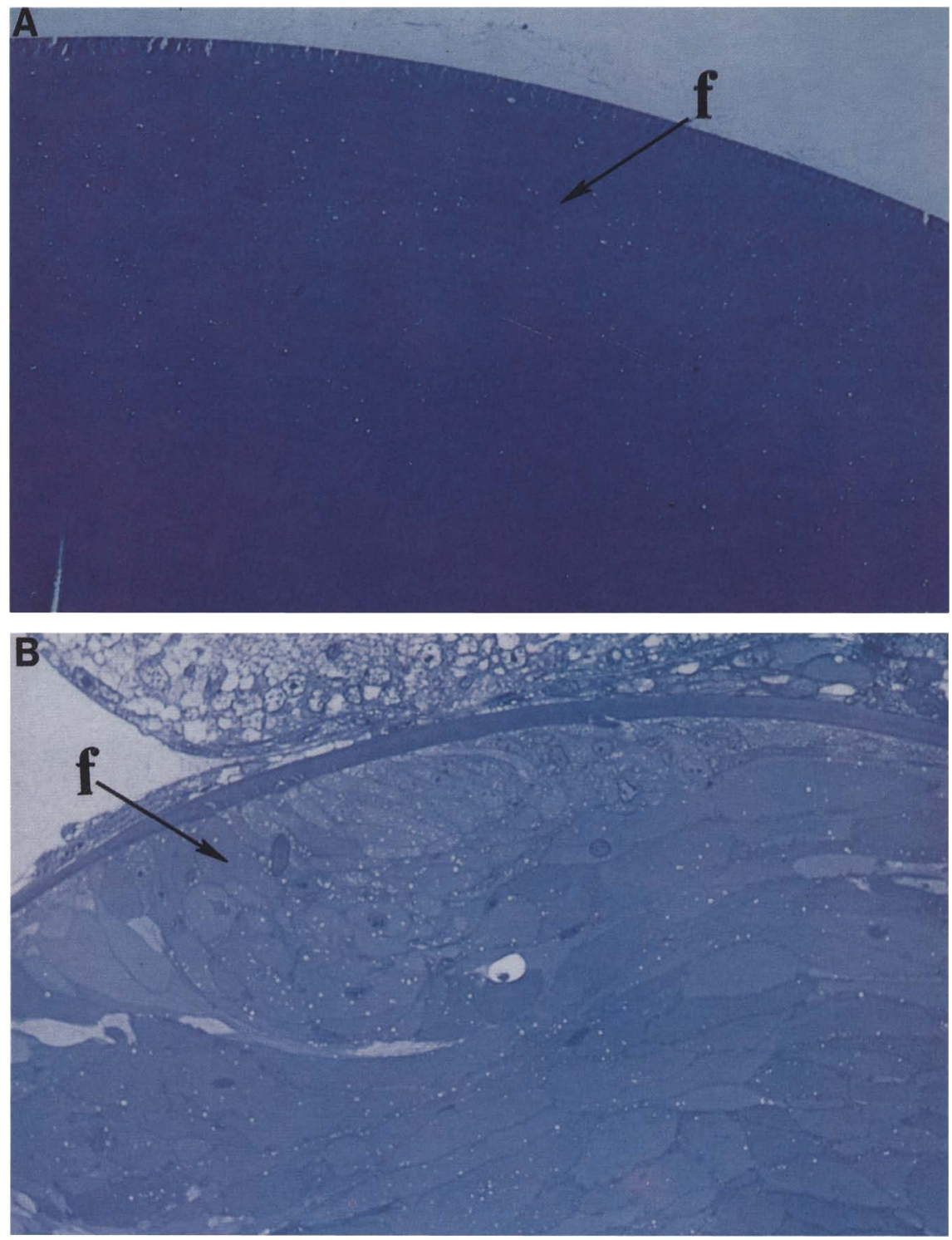

Figure 5. Aberrant development of lens in transgenic mice expressing the $\alpha \mathrm{A}$-crystallin/ricin A fusion gene. Normal $(A)$ and transgenic $(B)$ lens. $|f|$ Lens fiber cells.

coupled with the data from transgenic animal experiments, implies that perturbation of lens development at day 10 or earlier results in severe retinal abnormalities, whereas perturbation at a later stage (Breitman et al. 1987) leads to normal retinal formation. These studies suggest that either naturally occurring mutations or directed toxin expression in transgenic mice inducing a failure of lens formation leads to an arrest of eye growth with subsequent retinal folding and malformation. This study allows a direct test of this model and suggests that the normal development of the lens is essential for the combined morphogenesis of the eye and correct orientation and development of the neurosensory retina. The approach of creating genetic alterations in which cell death and subsequent developmental arrest can be programmed precisely should allow a unique approach to a number of significant questions in developmental biology.

\section{Experimental procedures}

\section{Hybrid gene construction}

The structure of the $\alpha \mathrm{A}$-crystallin/ricin A hybrid gene is shown in Figure 1. A 935-nucleotide XhoI-Sall fragment encoding $R$. communis preproricin from amino acid -24 to 285 was purified from plasmid pUC8RA, a pUC8 subclone of plasmid pRCL6 (Lamb et al. 1985), and used to construct hybrid genes for toxic ablation experiments. This fragment contains the entire ricin A chain sequence (up to and including amino acid 267) 

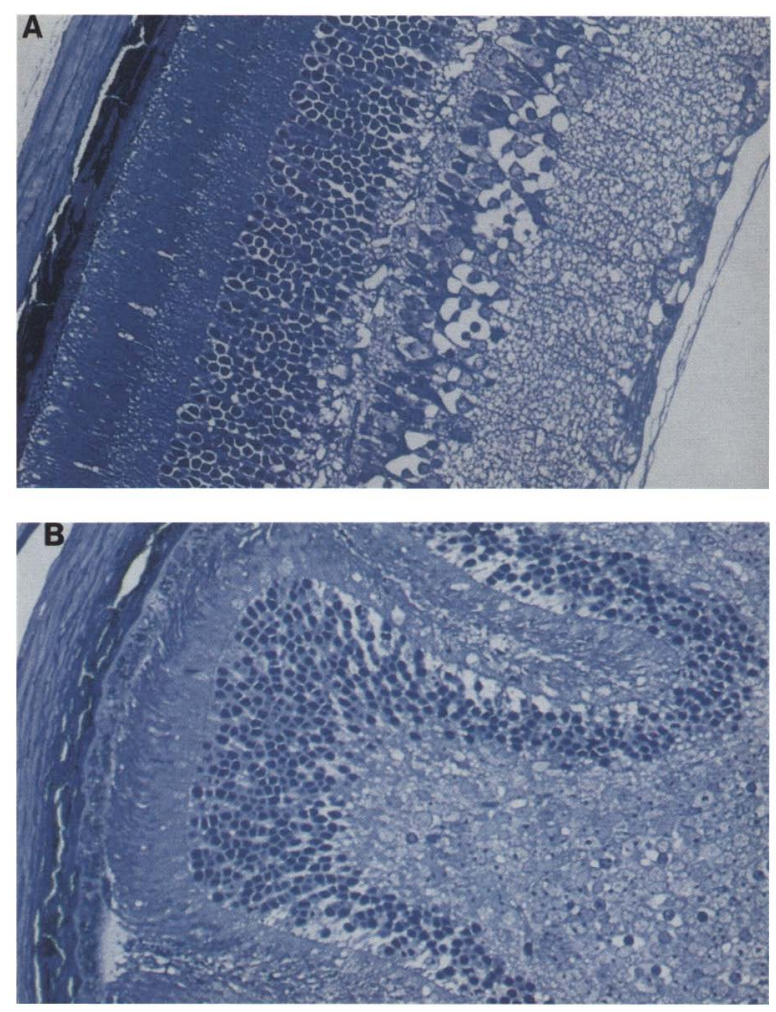

Figure 6. Aberrant structure of the retina in transgenic mice expressing ricin A. Retinal morphology of a normal control $(A)$ mouse and transgenic $(B)$ animal.

and 18 amino acids of additional sequence representing the connecting peptide and the amino-terminal portion of the ricin B chain. The $X$ hoI site was supplied by a synthetic oligonucleotide beginning one nucleotide upstream of the initiator ATG, while the $11 \mathrm{bp}$ downstream of the amino-terminal methionine codon, from the termination codon to the Sall site, were supplied by the pUC8 vector into which the preproricin fragment was originally cloned. This fragment was cloned into the Sall site of plasmid pUC19. A 416-nucleotide, BglII-BamHI fragment containing the promoter, transcriptional initiation site, and 367 nucleotides of $5^{\prime}$ flanking sequence of the mouse $\alpha \mathrm{A}$ crystallin gene was removed from plasmid pMaAcr 1800 (Chepelinsky et al. 1985) and cloned into the BamHI site in the pUC19 linker, located $5^{\prime}$ of the ricin A gene. This plasmid was then digested with HindIII, and a 24-nucleotide synthetic oligomer (AGCTTAATAAACCCGGGTTTATTA) was added to the $3^{\prime}$ end of the ricin A gene to supply a poly(A) addition signal and a SmaI site. The resulting plasmid carrying the $\alpha \mathrm{A}$-crystallin/ricin A fusion gene, pCRASA, was digested with SmaI, separating the entire hybrid gene from the plasmid vector, and the restriction fragment was purified on an agarose gel prior to microinjection into isolated mouse embryos.

\section{Production of transgenic mice}

Transgenic mice were produced as described previously (Chen et al. 1987) by microinjecting DNA at a concentration of 1 $\mu \mathrm{g} / \mathrm{ml}$ into the male pronucleus of isolated CB6F2/J zygotes. Embryos were then reimplanted into pseudopregnant CD-1 female hosts according to methods published previously (Hogan et al. 1986). Transgenic mice were identified by Southern blot hybridization of DNA isolated from tails using probes specific for the $\alpha$ A-crystallin or ricin A portions of the transgene (Fig. 1). Hybridization probes were purified from low melting point agarose gels and labeled to a sp. act. of $1 \times 10^{8}$ to $3 \times 10^{8} \mathrm{cpm} /$ $\mu \mathrm{g}$ by random hexamer-primed synthesis (Feinberg and Vogelstein 1983).

\section{Histological analysis}

Mice were anesthetized with avertin, and one or both eyes enucleated. Eyes were fixed by immersion of $1 \%$ glutaraldehyde in $0.1 \mathrm{M}$ sodium phosphate buffer $(\mathrm{pH} 7.2)$ and stored in $2 \%$ formaldehyde for 1 week. They were then fixed for $1 \mathrm{hr}$ in $1 \% \mathrm{OsO}_{4}$,
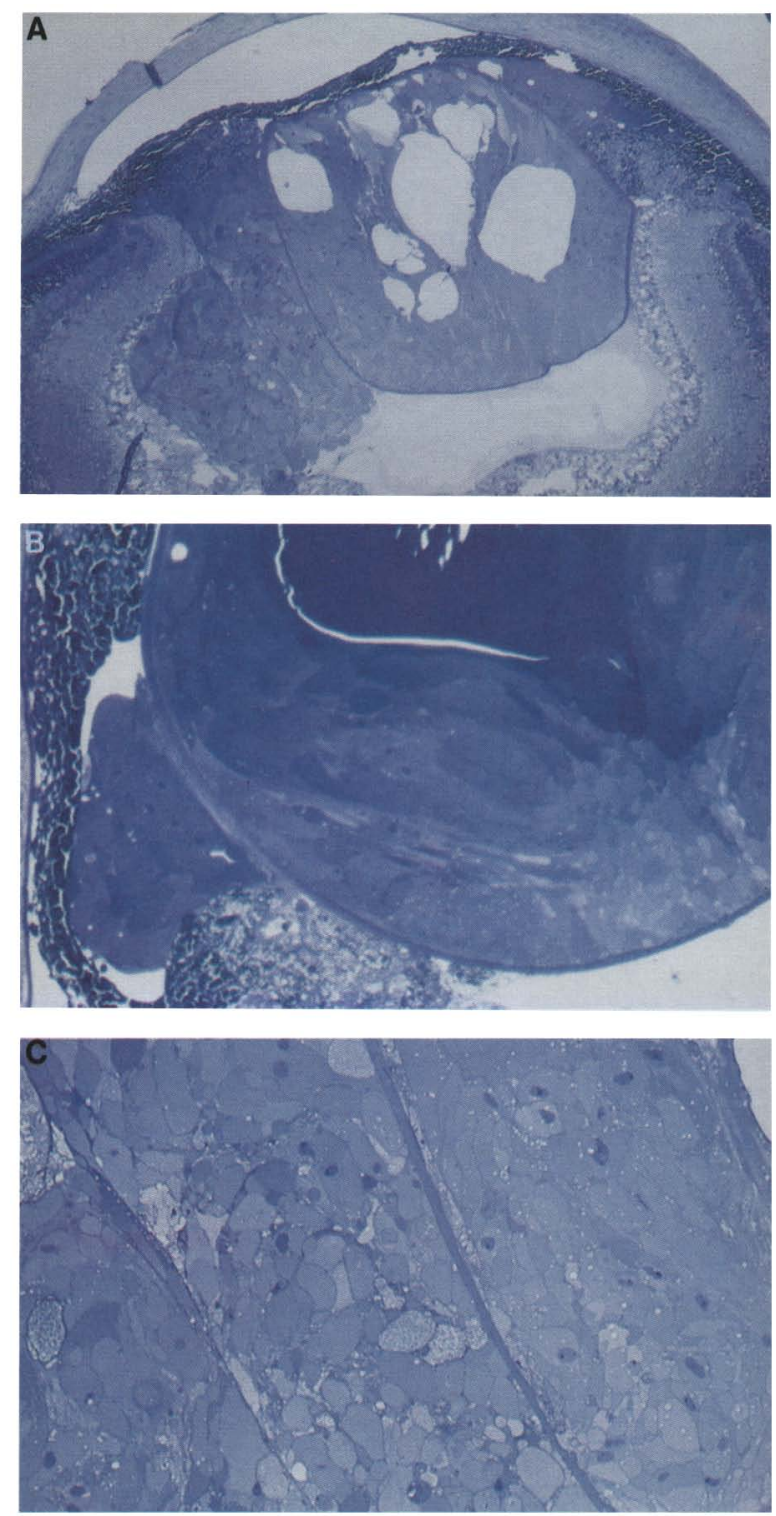

Figure 7. Ectopic lens fiber cells in transgenic eyes. Ectopic material can be seen outside of the lens capsule, laterally to the lens in two different individuals $(A, B)$. In close-up $(C)$, the morphology of the ectopic cells $(\mathrm{ec})$ is very similar to that of the fiber cells (fc) inside the lens capsule (arrows). 
embedded in Araldite 502, sectioned on a microtome, using glass knives, and stained with toluidine blue.

\section{Acknowledgments}

We thank Drs. D. Schubert, J. Piatigorsky, J.M. Lord, and L. Roberts for plasmids; R. Heyman and R. Evans for discussions of unpublished results; G. Andreason, S. Chen, J. Eubanks, G.M. Lawless, K. Lewis, K. Pischel, B.E. Rothenberg, S. LeVay, and $\mathrm{L}$. Swanson for advice and helpful discussions; and A. Wurster and A. Albi for assistance. This work was supported by grants to G.A.E. and D.B. from the National Institutes of Health, the March of Dimes Birth Defects Foundation, the Hereditary Disease Foundation, and the G. Harold and Leila Y. Mathers Charitable Foundation. G.A.E. is a Pew Scholar in the Biomedical Sciences supported by the Pew Charitable Trust. D.B. is Dolly Green Professor of Ophthalmology and Research to Prevent Blindness Senior Scientific Investigator.

\section{References}

Bloemendal, H. 1982. Lens proteins. Crit. Rev. Biochem. 12: $1-38$.

Breitman, M.L., S. Clapoff, J. Rossant, L.-C. Tsui, L.M. Glode, I.H. Maxwell, and A. Bernstein. 1987. Genetic ablation: Targeted expression of a toxin gene causes microphthalmia in transgenic mice. Science 238: 1563-1565.

Burns, R.S., C.L. Chiueh, S.P. Markey, M.H. Ebert, D.M. Jacobwitz, and I.J. Kapin. 1983. A primate model for parkinsonism: Selective destruction of dopaminergic neurons in the pars compacta of the substantia nigra by N-methyl-4phenyl-1, 2, 3, 6-tetrahydropyridine. Proc. Natl. Acad. Sci. 80: $4546-4550$.

Chen, S., F. Botteri, H. van der Putten, C.P. Landel, and G.A. Evans. 1987. A lymphoproliferative abnormality associated with inappropriate expression of the Thy-1 antigen in transgenic mice. Cell 51: 7-19.

Chepelinsky, A.B., B. Sommer, and J. Piatigorsky. 1987. Interaction between two different regulatory elements activates the murine $\alpha$ A-crystallin gene promoter in explanted lens epithelia. Mol. Cell. Biol. 7: 1807-1814.

Chepelinsky, A.B., C.R. King, P.S. Zelenka, and J. Piatigorsky. 1985. Lens-specific expression of the chloramphenicol acetyltransferase gene promoted by $5^{\prime}$ flanking sequences of the murine $\alpha$ A-crystallin gene in explanted chicken lens epithelia. Proc. Natl. Acad. Sci. 82: 2334-2338.

Columbre, A.J. and J.L. Columbre. 1964. Lens development. I. Role of the lens in eye growth. J. Exp. Zool. 156: 39-48.

Endo, Y. and K. Tsurugi. 1987. RNA n-glycosidase activity of the ricin A chain. I. Biol. Chem. 262: 8128-8130.

Feinberg, A.P. and B. Vogelstein. 1983. A technique for radiolabeling DNA restriction endonuclease fragments to high specific activity. Anal. Biochem. 132: 6-13.

Herrup. K. 1986. Cell lineage relationships in the development of the mammalian CNS: Role of cell lineage in the control of cerebellar Purkinje cell number. Dev. Biol. 115: 148-154.

Hogan, B., F. Constantini, and E. Lacy, 1986. Manipulating the mouse embryo. Cold Spring Harbor Laboratory, Cold Spring Harbor, New York.

Horvitz, H.R., P.W. Sternberg, I.S. Greenwald, W. Fixsen, and H.M. Ellis. 1983. Mutations that affect neural cell lineages and cell fates during the development of the nematode Caenorhabditis elegans. Cold Spring Harbor Symp. Quant. Biol. 48: 453-463.
Jacobsen, M. 1981. Rohon-Beard neurons originate from blastomeres of the 16-cell frog embryo. J. Neurosci. 1: 918-922.

Kimmel, C.B. and R.M. Warga. 1986. Tissue-specific cell lineages originate in the gastrula of the zebrafish. Science 231: 365-368.

King, C.R. and J. Piatigorski. 1983. Alternative RNA splicing of the murine $\alpha \mathrm{A}$-crystallin gene: Protein-coding information within an intron. Cell 32: 707-712.

Lamb, F.I., L.M. Roberts, and M.J. Lord. 1985. Nucleotide sequence of cloned cDNA coding for preproricin. Eur. J. Biochem. 148: 365-270.

Mahon, K.A., A.B. Chepelinsky, J.S. Khillan, P.A. Overbeek, J. Piatigorsky, and H. Westphal. 1987. Oncogenesis of the lens in transgenic mice. Science 235: 1622-1628.

Okada, T.S. 1980. Cellular metaplasia or transdifferentiation as a model for retinal cell differentiation. Curr. Top. Dev. Biol. 16: 349-390.

1983. Recent progress in studies of the transdifferentiation of eye tissue in vitro. Cell Differ. 13: 177-183.

Olsnes, S. and A. Phil. 1982. Toxic lectins and related proteins. In Molecular actions of toxins and viruses (ed. P. Cohen and S. Van Heyningen), pp. 51-105. Elsevier Biomedical Press, Amsterdam.

Overbeek, P.A., A.B. Chepelinsky, J.S. Khillan, J. Piatigorsky, and $H$. Westphal. 1985. Lens-specific expression and developmental regulation of the bacterial chloramphenicol acetyltransferase gene driven by the murine $\alpha \mathrm{A}$-crystallin promoter in transgenic mice. Proc. Natl. Acad. Sci. 82: 78157819.

Palmiter, R.D., R.R. Behringer, C.J. Quaife, F. Maxwell, I.H. Maxwell, and R.L. Brinster. 1987. Cell lineage ablation in transgenic mice by cell-specific expression of a toxin gene. Cell 50: 435-443.

Pastan, I., M.C. Willingham, D.J.P. FitzGerald. 1986. Immunotoxins. Cell 47: 641-648.

Price, J., D. Turner, and C. Cepko. 1987. Lineage analysis in the vertebrate nervous system by retrovirus-mediated gene transfer. Proc. Natl. Acad. Sci. 84: 156-160.

Reh, T.A., T. Nagy, and H. Gretton. 1987. Retinal pigmented epithelial cells induced to transdifferentiate to neurons by laminin. Nature 330: $68-71$.

Roux, W. 1888. Beitrage zur Entwickelungsmechanik des Embryo. V. Ueber die kunstliche Hervorbringung 'halber' Embryonen durch Zerstorung einer der beiden ersten Furchungzellen, sowie uber die NachJentwickelung (Postgeneration) der fehlenden Korperhalfte. Virchows Arch. Pathol. Anat. Physiol. 114: 113-153; 246-291.

Sulston, J.E., and J.G. White. 1980. Regulation and cell autonomy during postembryonic development of Caenorhabditis elegans. Dev. Biol. 56: 110-156.

Turner, D.L. and C.L. Cepko. 1987. A common progenitor for neurons and glia persists in rat retina late in development. Nature 328: $131-136$.

Van Leen, R.W., K.E.P. Van Roozendaal, N.H. Lubsen, and J.G.G. Schoenmakers. 1987a. Differential expression of crystallin genes during development of the rat eye lens. Dev. Biol. 120: 457-464.

Van Leen, R.W., M.L. Breuer, N.H. Lubsen, and J.G.G. Schoenmakers. 1987b. Developmental expression of crystallin genes: In situ hybridization reveals a differential localization of specific mRNAs. Dev. Biol. 123: 338-345.

Varnum, D.S. and L.C. Stevens. 1968. Aphakia, a new mutation in the mouse. J. Hered. 59: 147-150.

Yasuda, K., K. Okuyama, and T.S. Okada. 1983. The accumulation of $\delta$-crystallin mRNA in transdetermination and trans- 


\section{Landel et al.}

differentiation of neural retina cells into lens. Cell Differ. 12: $177-183$.

Zwaan, I. 1975. The appearance of $\alpha$ A-crystallin in relation to cell cycle phase in the embryonic mouse lens. Dev. Biol. 96: $173-181$.

Zwaan, J. and B.M. Kirkland. 1975. Malorientation of mitotic figures in the early lens rudiment of aphakia mouse embryos. Anat. Rec. 182: 345-354.

Zwaan, J. and J. Silver. 1983. Crystallin synthesis in the lens rudiment of a strain of mice with congenital anophthalmia. Exp. Eye Res. 36: 551-557. 


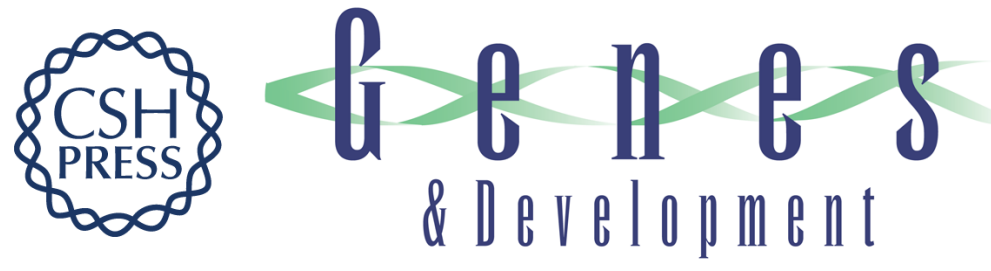

\section{Lens-specific expression of recombinant ricin induces developmental defects in the eyes of transgenic mice.}

C P Landel, J Zhao, D Bok, et al.

Genes Dev. 1988, 2:

Access the most recent version at doi:10.1101/gad.2.9.1168

References This article cites 33 articles, 11 of which can be accessed free at:

http://genesdev.cshlp.org/content/2/9/1168.full.html\#ref-list-1

License

Email Alerting

Service

Receive free email alerts when new articles cite this article - sign up in the box at the top right corner of the article or click here.

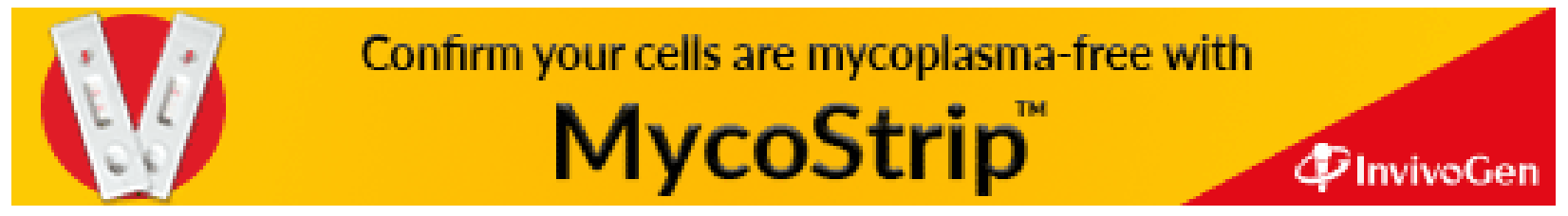

